\title{
PROJECTIVE MODULES OVER RINGS WITH MANY UNITS
}

\author{
B. R. MCDONALD AND WILLIAM C. WATERHOUSE ${ }^{1}$
}

\begin{abstract}
Let $R$ be a commutative ring. Assume that every polynomial whose values generate the unit ideal actually takes on an invertible value. Then projective $\boldsymbol{R}$-modules split into cyclic summands, and those of constant rank are free.
\end{abstract}

A ring $R$ (commutative with 1) satisfies the primitive condition if each $f(x)=a_{0}$ $+\cdots+a_{n} X^{n}$ that is primitive $\left(\Sigma\left(a_{i} R\right)=R\right)$ has some $b$ in $R$ with $f(b)$ a unit. This condition, which guarantees the existence of many units in $R$, was introduced by van der Kallen [13]; he gave examples of rings satisfying the condition and established properties of $K_{2}(R)$ for such $R$. Subsequently it was shown [4], [5], [6] that the condition implies pleasant structural results about $\mathrm{GL}_{2}(R)$ and $\operatorname{Aut}\left(\mathrm{GL}_{2}(R)\right)$. One step in this was a computational argument [6, II.3] proving that if $Q$ is a rank one direct summand of $F$ where $F$ is free of rank 2 , then $Q$ is free. We here will see that a much more general result is true. It actually applies to a slightly larger class of rings, and we begin by discussing them.

I. Let $f\left(X_{1}, \ldots, X_{n}\right)$ be a polynomial over a ring $R$. We will say that $f$ has local unit values if for each maximal ideal $M$ of $R$ there are $b_{1}, \ldots, b_{n}$ in $R_{M}$ with $f\left(b_{1}, \ldots, b_{n}\right)$ invertible in $R_{M}$. We can here replace the $b_{i}$ by elements of $R$ congruent to them modulo $M$, so the condition says that not all values of $f$ are in $M$; in other words, the values of $f$ should generate the unit ideal of $R$. (This implies, of course, that the coefficients of $f$ must generate the unit ideal.) We say that $f$ has unit values if some $f\left(b_{1}, \ldots, b_{n}\right)$ is actually invertible in $R$.

The rings we care about will be those in which every $f$ with local unit values has unit values. Since elements are invertible iff they are so modulo the Jacobson radical $J(R)$, it is evident that $R$ has this property iff $R / J(R)$ does. In particular, semilocal rings have the property. It is also easy to see that a product $\Pi R_{i}$ has the property iff all the factors do (consider maximal ideals of the form $M_{i} \times \Pi_{j \neq i} R_{j}$ ). Further examples of rings with this property are given by the following propositions.

Proposition. Let $R$ be a ring for which $R / J(R)$ is von Neumann regular (= absolutely flat). Then polynomials with local unit values have unit values.

Proof. Replacing $R$ by $R / J(R)$, we may assume it is von Neumann regular. Suppose $f$ has local unit values. For each maximal $M$ pick $b=\left(b_{1}, \ldots, b_{n}\right)$ with

Received by the editors August 28, 1980.

1980 Mathematics Subject Classification. Primary 13 C05.

${ }^{1}$ The work of both authors was supported in part by the National Science Foundation. 
$f(b)$ a unit at $M$; then $f(b)$ is still a unit on a neighborhood of $M$ in Spec $R$. Since Spec $R$ is a Boolean space, we can refine this covering to a finite covering by disjoint clopen sets $U$ where we have $f\left(b_{U}\right)$ invertible on $U$. We may then choose $b$ agreeing with $b_{U}$ on $U$, and $f(b)$ will be invertible. $\square$ This result could also be deduced from [2, Proposition 2].

The argument shows more generally that if we have a sheaf of rings over a Boolean space and the fibers have our property, so does the ring of global sections. We should also point out that by [1, II.4, Exercise 16, p. 173] we have the following special case of the proposition:

COROllary. Let $R$ be zero-dimensional. Then polynomials with local unit values have unit values.

PROPOSITION. Let $S$ be an $R$-algebra which is a finitely generated free $R$-module. Suppose that over $R$, all polynomials with local unit values have unit values. Then the same is true over $S$.

Proof. Let $s_{1}, \ldots, s_{m}$ be a basis of $S$ over $R$. Given $f\left(X_{1}, \ldots, X_{n}\right)$ over $S$, take indeterminates $Y_{11}, \ldots, Y_{m n}$ and define a polynomial $g(Y)$ over $R$ as the norm (from $S$ to $R$ ) of $f\left(\sum s_{i} Y_{i 1}, \ldots, \Sigma s_{i} Y_{i n}\right)$. Then setting $X_{j}=\sum s_{i} r_{i j}$ makes $f(X)$ invertible iff $g\left(r_{i j}\right)$ is invertible, since units and only units have unit norms. Assume now that $f$ has local unit values. If $M$ is any maximal ideal of $R$, then $S_{M}$ is semilocal, so $f$ has unit values in $S_{M}$. Hence $g$ has unit values in $R_{M}$. By hypothesis then $g$ has unit values in $R$. $\square$ The main theorem will automatically allow us to replace "free" by "projective of constant rank" in this result.

We now show exactly how our property is related to the primitive condition mentioned in the introduction. For this we need a pair of simple lemmas.

LEMMA. Let $R$ satisfy the primitive condition. Let $f_{i}(X)=\sum a_{i j} X^{j}$ be a finite sequence of polynomials with $\Sigma_{i, j}\left(a_{i j} R\right)=R$. Then there is some $b$ in $R$ with $\Sigma\left(f_{i}(b) R\right)=R$.

Proof. Choose an integer $m$ greater than the degrees of all $f_{i}$, and let $g(X)=$ $\sum f_{i}(X) X^{m i}$. All $a_{i j}$ occur as coefficients in $g$, so $g$ is primitive. Hence some $g(b)=\sum f_{i}(b) b^{m i}$ is a unit, and in particular $\Sigma\left(f_{i}(b)\right) R=R$.

This allows us to deduce a multivariable extension of the condition:

Lemma. Let $R$ satisfy the primitive condition. Let $f\left(X_{1}, \ldots, X_{n}\right)=\sum a_{\alpha} X^{\alpha}$ be a polynomial with $\Sigma\left(a_{\alpha} R\right)=R$. Then there are $b_{1}, \ldots, b_{n}$ in $R$ with $f\left(b_{1}, \ldots, b_{n}\right)$ invertible.

Proof. Rewrite $f$ as $\Sigma_{\beta} f_{\beta}\left(X_{1}\right) X^{\beta}$, where $\beta=\left(i_{2}, \ldots, i_{n}\right)$. All the $a_{\alpha}$ appear as the coefficients of the polynomials $f_{\beta}\left(X_{1}\right)$. By the lemma there is some $b_{1}$ such that $\Sigma\left(f_{\beta}\left(b_{1}\right) R\right)=R$. Then $f\left(b_{1}, X_{2}, \ldots, X_{n}\right)$ again satisfies the hypothesis of the lemma, and the result follows by induction.

PROPOSITION. A ring $R$ satisfies the primitive condition iff

(1) every polynomial with local unit values has unit values and

(2) every residue field $R / M$ is infinite. 
Proof. If $R / M$ has finite cardinality $q$, then $X^{q}-X$ is a primitive polynomial with all values in $M$. Thus if $R$ satisfies the primitive condition, (2) must hold. And any $f\left(X_{1}, \ldots, X_{n}\right)$ with local unit values has coefficients generating the unit ideal, so (1) holds by the last lemma. Conversely, if $f$ is a primitive polynomial, then it is nontrivial modulo $M$, so by (2) it has a nonzero value modulo $M$. This means it has local unit values, so by (1) it has unit values.

This shows in particular that rings with our property can be very far from dimension zero. Indeed [3], if $A$ is any ring and $S$ is the set of primitive polynomials in $A[x]$, then $R=S^{-1} A[x]$ satisfies the primitive condition and has maximal ideal space identical with that of $A$.

II.

THEOREM. Let $R$ be a ring over which every polynomial with local unit values has unit values. Let $P$ be a finitely generated projective $R$-module of constant rank. Then $P$ is free.

Proof. For some $n$ we can write $R^{n}=P \oplus Q$. Let $A$ be the matrix of the map $R^{n} \rightarrow R^{n}$ that projects onto $P$ with kernel $Q$. Let $X=\left(X_{i j}\right)$ be a matrix of indeterminates and let $X^{c}$ be the matrix of cofactors, so that $X X^{c}=(\operatorname{det} X) I$. Let $m$ be the (constant) local rank of $P$, and let $g\left(X_{i j}\right)$ then be the polynomial which is the upper left $m \times m$ minor determinant of $X A X^{c}$. Set $f\left(X_{i j}\right)=g\left(X_{i j}\right) \operatorname{det}\left(X_{i j}\right)$.

Let $M$ be a maximal ideal of $R$. Over the local ring $R_{M}$, both $P_{M}$ and $Q_{M}$ are free, with $P_{M}$ of rank $m$. Choose a basis of $R_{M}^{n}$ compatible with the decomposition $P_{M} \oplus Q_{M}$. In this basis the projection to $P_{M}$ has matrix

$$
\left(\begin{array}{cc}
I_{m} & 0 \\
0 & 0
\end{array}\right)
$$

Thus if $\left(c_{i j}\right)$ is the matrix in $R_{M}$ giving the change of basis, $g\left(c_{i j}\right)=\left(\operatorname{det}\left(c_{i j}\right)\right)^{m}$. Hence $f\left(c_{i j}\right)$ is a unit in $R_{M}$. Since this is true for every maximal $M$, there are values $b_{i j}$ in $R$ with $f\left(b_{i j}\right)$ invertible. Then the matrix $\left(b_{i j}\right)$ is invertible, and the upper left $m \times m$ submatrix of $D=\left(b_{i j}\right) A\left(b_{i j}\right)^{-1}$ is invertible.

The rest is routine. Make the base change in $R^{n}$ given by $\left(b_{i j}\right)$, so that $D$ becomes the matrix of the projection onto $P$. Let $F=R^{m} \times 0$, and let $p$ be the projection $R^{n} \rightarrow F$ with kernel $0 \times R^{n-m}$. Then $F \rightarrow R^{n} \rightarrow^{D} R^{n} \rightarrow^{P} F$ has invertible matrix. Consequently the map from $P=D\left(R^{n}\right)$ to $F$ via $p$ is surjective. Since $F$ is free we have $P \simeq F \oplus K$ for some $K$. Both $F_{M}$ and $P_{M}$ have rank $m$ over $R_{M}$, so $K_{M}=0$ for all maximal ideals $M$. Hence $K=0$ and $P \simeq R$.

COROLlary. The Picard group of $R$ is trivial.

This corollary has implications related to [6]. Specifically, it implies [7, §1] that for every $n$ the "standard" automorphisms of $\mathrm{GL}_{n}(R)$ induced by $\mathrm{PGL}_{n}(R)$ are actual inner automorphisms of $\mathrm{GL}_{n}(R)$.

COROLlaRY. Any finitely generated projective $R$-module is a direct sum $\bigoplus \mathbf{R e}_{i}$ where the $e_{i}$ are idempotents of $R$. 
Proof. If $P$ is such a module, then its rank is a locally constant function on Spec $R$, by [1, II.5.3, Theorem 2, p. 141]. Correspondingly then we can split $R$ into a product $\operatorname{Re}_{1} \times \cdots \times \operatorname{Re}_{n}$ so that $P=\bigoplus\left(e_{i} P\right)$ has each $e_{i} P$ of constant rank over $\operatorname{Re}_{i} . \mathrm{But} \mathrm{Re}_{i}$ is a direct factor of $R$ and hence also satisfies the hypothesis, so the theorem applies to $e_{i} P$.

Note added March 16, 1981. D. R. Estes and R. M. Guralnick have now made a more extensive study of rings $R$ for which polynomials with local unit values have unit values. Among other things they generalize our theorem by showing that arbitrary finitely presented $R$-modules $P$ and $Q$ are isomorphic whenever $P_{M} \simeq$ $Q_{M}$ for all maximal $M$.

\section{REFERENCES}

1. N. Bourbaki, Algèbre commutative, Chapters I, II, Hermann, Paris, 1961.

2. K. R. Goodearl and R. B. Warfield, Jr., Algebras over zero-dimensional rings, Math. Ann. 223 (1976), 157-168.

3. W. van der Kallen, The $K_{2}$ of rings with many units, Ann. Sci. École Norm. Sup. (4) 10 (1977), 473-515.

4. B. R. McDonald, $\mathrm{GL}_{2}$ of rings with many units, Comm. Algebra 8 (1980), 869-888.

5. __ Projectivities over rings with many units, Comm. Algebra 9 (1981), 195-204.

6.

7. W. C. Waterhouse, Automorphisms of $\mathrm{GL}_{n}(R)$, Proc. Amer. Math. Soc. 79 (1980), 347-351.

Department of Mathematics, University of OxLahoma, Norman, OrLahoma 73019

Department of Mathematics, Pennsylvania State University, University Park, Pennsylvania 16802 